\title{
Design of the multi-reflection time-of-flight mass spectrometer for the RAON facility
}

\author{
J. W. Yoon ${ }^{1, \text { a }}$, Y. -H. Park ${ }^{1}$, S. J. Park ${ }^{1}$, G. D. Kim ${ }^{1}$, and Y. K. Kim¹,2 \\ ${ }^{1} R I S P$, Institute of Basic Science, Daejeon, Republic of Korea \\ ${ }^{2}$ Department of Nuclear Engineering, Hanyang University, Seoul, Republic of Korea
}

\begin{abstract}
Electrodes design for the multi-reflection time-of-flight mass spectrometer (MR-TOF-MS) has been performed for the high precision mass measurement system in RAON accelerator facility, which will be constructed in Korea. Calculated mass resolution is $10^{5}$ for the condition of an energy spread of $\pm 30 \mathrm{eV}$ and an emittance of 0.75 $\pi \mathrm{mm}$ mrad. MR-TOF-MS will be used for the isobar separation and the mass measurement for very short-lived isotopes.
\end{abstract}

\section{Introduction}

The Rare Isotope Science Project (RISP) for constructing the heavy ion accelerator called RAON was launched in Korea in 2011. RAON is a pure Korean word, meaning delightful. The project aims to construct the new accelerator complex including the Isotope Separation On-Line (ISOL) facility and the In-Flight Fragment (IF) facility. In the RAON complex, the high precision mass measurement system will be constructed to offer precise mass value of rare isotopes for understanding nuclear structure. Presently, the Penning trap mass spectrometer (PTMS) provides the most precise means for measuring atomic masses $[1,2]$. In order to improve mass precision of PTMS, highly charged ions (HCI) have recently introduced to the PTMS [3-6], since mass precision scales with the charge state q. In the RAON mass measurement system, a PTMS employing HCI will be deployed to achieve mass accuracy of less than $10^{-8}$. However, PTMS is not suitable for very short-lived nuclei $[7,8]$, since PTMS requires measurement times in excess of $100 \mathrm{~ms}$. For the fast, high precision mass measurements, we will develop another device, a multi-reflection time-of-flight mass spectrometer (MR-TOF-MS), which allows to achieve a very high mass resolving power $\left(>10^{5}\right)$ with extremely short measurement times (several ms) in a compact device. MR-TOF-MS has been proposed by Wollnik in 1990 [9] and developed at RIBF/RIKEN [7, 8], at SHIPTRAP/GSI [10-12], and at ISOLTRAP/CERN [13-18], etc. Recently, MR-TOF-MS has been commissioned online at ISOLTRAP/CERN for the first time [15-18]. MR-TOF-MS can serve not only as devices for high-accuracy mass measurements of very short-lived nuclei, but also as isobar separators [11, 16].

To minimize uncertainties in PTMS, contamination in the ions should be removed, since contaminations would change the motion of the ions of interest. To remove the contaminating ions, resonant buffer-gas cooling is usually used [19], but HCI cannot be purified in the buffer-gas filled preparation

\footnotetext{
a e-mail: yoonjw@ibs.re.kr
} 
trap due to charge exchange processes. Therefore, an MR-TOF-MS acts also as an isobar separator for the PTMS employing HCI [4].

In this paper, simulation results for the MR-TOF-MS design using SIMION code are presented. Temporal broadenings, caused by the kinetic energy spread and the transverse emittance, were minimized by optimization of the electrode potentials, and it was demonstrated that the mass resolving power of $10^{5}$ is achievable.

\section{Design of the MR-TOF-MS}

The MR-TOF-MS is composed of two electrostatic ion mirrors in combination with einzel lenses. Ions are cooled and bunched by RF ion cooler/buncher, and then bunched ions enter the MR-TOFMS, when the mirror electrode voltages are switched off. The injected ions travel for hundreds of revolutions, and during oscillations ions with different masses are temporally separated. When temporal separation becomes larger than the ion bunch width $(\Delta T)$, ions are extracted from the MR-TOF-MS by switching off the mirror voltages, and then arrive at a detector plane located at time focus, where an MCP detector for the mass measurement or an ion gate for the isobar separation is deployed [10]. The ion gate transmits the ions of interest, but deflects all other ions. Mass resolving power of the MR-TOF-MS is given approximately by equation (1) [13], where the total round trip time is denoted as $T_{\text {total }}$, initial bunch width as $\Delta T_{\text {bunch }}$, and temporal broadenings, due to the energy spread and the transverse emittance, as $\Delta T_{\text {energy }}$ and $\Delta T_{\text {emittance }}$, respectively.

$$
R=\frac{m}{\Delta m}=\frac{T_{\text {total }}}{2 \Delta T}=\frac{T_{\text {total }}}{2 \sqrt{\left(\Delta T_{\text {bunch }}\right)^{2}+\left(\Delta T_{\text {energy }}\right)^{2}+\left(\Delta T_{\text {emittance }}\right)^{2}}}
$$

To obtain high resolving power, $\Delta T_{\text {energy }}$ and $\Delta T_{\text {emittance }}$ should be minimized by optimization of the potential distribution in the ion mirrors [20]. For the MR-TOF-MS design, we used SIMION8.1 code and referred to the geometry of the MR-TOF-MS at ISOLTRAP (ISOLDE/CERN) [14].

Figure 1 shows the cross sectional view of the MR-TOF-MS, and figure 2 (a) shows the dimension of the mirror electrodes. In the RAON mass measurement system, the mass of doubly-magic ${ }^{132} \mathrm{Sn}$ nuclide will be measured at first, since tin isotopes near $A=132$ are planned to be the first RI beams extracted from the RAON ISOL facility. Therefore, electrode potentials were optimized for the ${ }^{132} \mathrm{Sn}^{+}$ ion with a kinetic energy $(K)$ of $1750 \mathrm{eV}$. Voltages applied to the electrodes are shown in table 1 , and the potential distribution in the electrodes is shown in figure 2 (b).

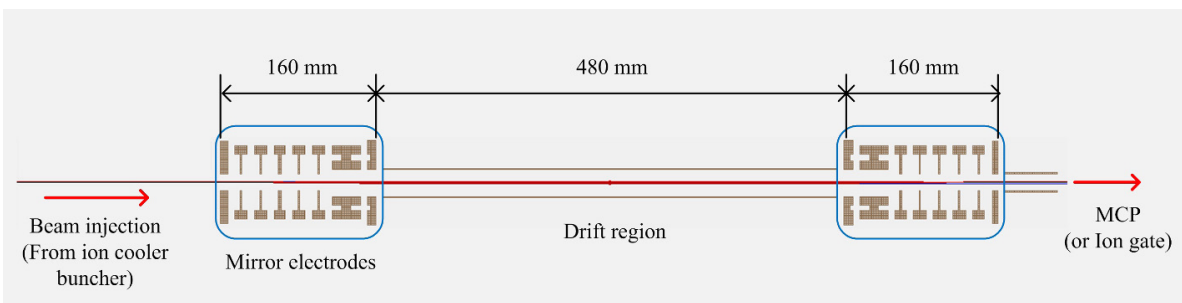

Figure 1. Cross sectional view of the MR-TOF-MS

For this potential distribution, $\Delta T_{\text {energy }}$ was calculated with respect to the number of round trips $(N)$ for the ions with $K=1750 \pm 30 \mathrm{eV}$. The calculation results are shown in figure 3(a), where $\Delta T$ 

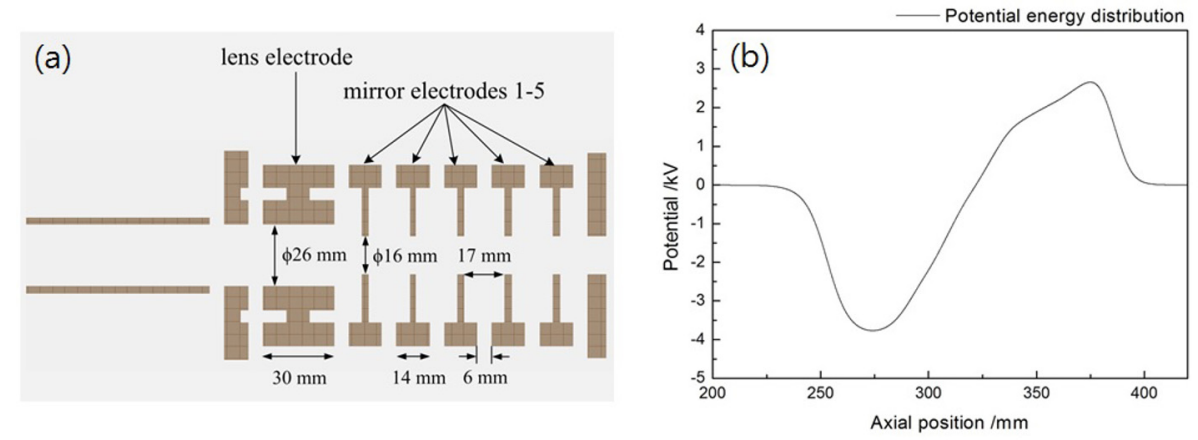

Figure 2. (a) Dimension of the mirror electrodes, (b) potential distribution in the mirror electrodes

Table 1. Voltages applied to the electrodes

\begin{tabular}{lllllll}
\hline Electrode & lens & mirror1 & mirror2 & mirror3 & mirror4 & mirror5 \\
\hline Voltage $[\mathrm{V}]$ & -3917.5 & -2300 & -230 & 1612.5 & 2152.5 & 3000 \\
\hline
\end{tabular}

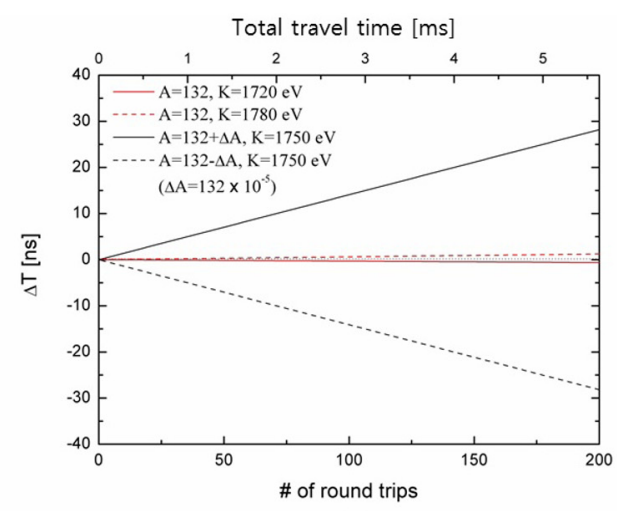

(a)

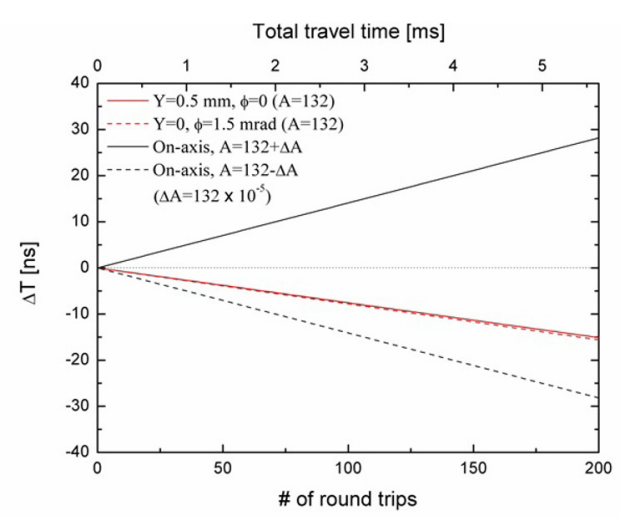

(b)

Figure 3. (a) Simulation results of temporal broadening due to the energy spread $(\Delta K= \pm 30 \mathrm{eV})$ with respect to the number of round trips, (b) simulation results of temporal broadening due to the emittance $(\epsilon=0.75 \pi \mathrm{mm}$ mrad) with respect to the number of round trips

is relative TOF to the reference ion with $K=1750 \mathrm{eV}$. We assume that all the ions are on axis, and start from the center of the MR-TOF-MS, where relative TOF is monitored every round trip. With increasing the number of round trips, the TOF difference between ions with different energy linearly increases. At $N=150\left(T_{\text {total }}=4.2 \mathrm{~ms}\right)$, temporal broadening is around $1.4 \mathrm{~ns}$. In addition, temporal spread due to mass difference of $\pm 10^{-5}$ is $42 \mathrm{~ns}$. Therefore, $\Delta T_{\text {energy }}$ is small enough to resolve mass difference of $\pm 10^{-5}$.

To investigate $\Delta T_{\text {emittance, }}$, we considered two ions on the vertexes of the emittance circle of $\epsilon=$ $0.75 \pi \mathrm{mm}$ mrad: one with $\phi=1.5 \mathrm{mrad}$ and $Y=0$, and the other with $\phi=0$ and $Y=0.5 \mathrm{~mm}$, where 
$\phi$ is the initial divergence angle and $Y$ is the initial vertical position from the axis. All the ions have same mass $(\mathrm{A}=132)$ and same kinetic energy $(K=1750 \mathrm{eV})$. Figure $3(\mathrm{~b})$ shows calculation results of temporal broadening due to transverse emittance, where $\Delta T$ is relative TOF to the on-axis reference ion $(\phi=0$ and $Y=0)$. In figure $3(\mathrm{~b})$, two ions show almost same TOF, and they are faster than on-axis reference ion. At $N=150\left(T_{\text {total }}=4.2 \mathrm{~ms}\right)$, temporal broadening is around $12 \mathrm{~ns}$. Therefore, considering the initial bunch width $\left(\Delta T_{\text {bunch }}\right)$ of $20 \mathrm{~ns}$ and $N=150$, mass resolving power of $1.1 \times 10^{5}$ can be obtained according to equation (1). In addition, same calculations were performed for the ions with different mass $(\mathrm{A}=8)$, and almost same mass resolving power $\left(10^{5}\right)$ was achieved for $T_{\text {total }}=4.2$ $\mathrm{ms}$. Therefore, mass resolving power for a given round trip time has no relation with ion mass.

\section{Conclusion}

We designed the MR-TOF-MS for the high precision mass measurement system in the RAON accelerator facility using SIMION code. For high resolving power, $\Delta T_{\text {energy }}$ and $\Delta T_{\text {emittance }}$ were minimized by optimization of the mirror electrode potential. Considering ${ }^{132} \mathrm{Sn}^{+}$ion with $K=1750 \mathrm{eV}(\Delta K= \pm 30$ $\mathrm{eV}), \Delta T_{\text {bunch }}=20 \mathrm{~ns}$, and $\epsilon=0.75 \pi \mathrm{mm} \mathrm{mrad}$, the resolving power of $10^{5}$ was achieved. This MRTOF-MS is expected to act as a high precision mass spectrometer and as an isobar separator at the RAON mass measurement facility.

\section{References}

[1] K. Blaum, Physics Reports 45, 1-78 (2006)

[2] H.-J. Kluge, Int. J. Mass Spectrom. 349-350, 26-37 (2013)

[3] I. Bergstöm, et al., Nucl. Instr. and Meth. in Phys. Res. A 487, 618-651 (2002)

[4] V.S. Kolhinen, et al., Nucl. Instr. and Meth. in Phys. Res. B 266, 4547-4550 (2008)

[5] J. Dilling, et al, Int. J. Mass Spectrom. 251, 198-203 (2006)

[6] S. Ettenauer, et al, Int. J. Mass Spectrom. 349-350, 74-80 (2013)

[7] Y. Ito, et al., Phys. Rev. C 88, 011306(R) (2013)

[8] P. Schury, et al., Nucl. Instr. and Meth. in Phys. Res. B 317, 537-543 (2013)

[9] H. Wollnik and M. Przewloka, Int. J. of. Mass Spectrom. Ion Proc. 96, 267-274 (1990)

[10] W.R. Plaß, et al., Nucl. Instrum. Meth. B 266, 4560-4564 (2008)

[11] W.R. Plaß, et al., Nucl. Instr. and Meth. in Phys. Res. B 317, 457-462 (2013)

[12] W.R. Plaß, et al., Int. J. Mass. Spectrom. 349-350, 134-144 (2013)

[13] R.N. Wolf, et al., Int. J. Mass Spectrom. 313, 8-14 (2012)

[14] R.N. Wolf, et al., Nucl. Instr. and Meth. in Phys. Res. A 686, 82-90 (2012)

[15] F. Wienholtz, et al., Nature 498 346-349 (2013)

[16] R.N. Wolf, et al., Phys. Rev. Lett. 110, 041101 (2013)

[17] S. Kreim, et al., Nucl. Instr. and Meth. in Phys. Res. B 317, $492-500$ (2013)

[18] R.N. Wolf, et al., Int. J. Mass Spectrom. 349-350, 123-133 (2013)

[19] G. Savard, et al., Phys. Lett. A 158, 247-252 (1991)

[20] H. Wollnik and A. Casares, Int. J. Mass. Spectrom. 227, 217-222 (2003) 